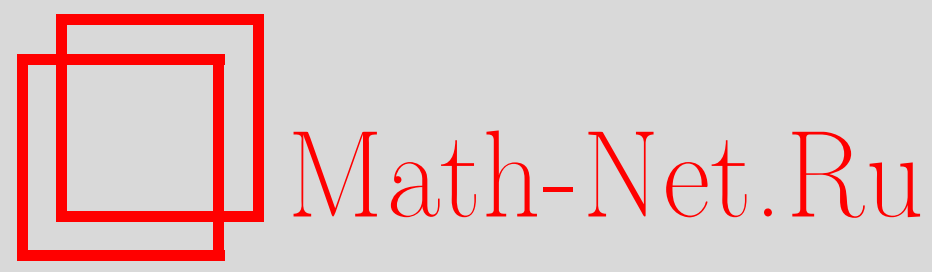

Д. В. Чистиков, О связи задач диагностического и проверяющего тестирования бесповторных функций, Дискрет. матем., 2011, том 23, выпуск 1, 46-50

DOI: https://doi.org/10.4213/dm1129

Использование Общероссийского математического портала Math-Net.Ru подразумевает, что вы прочитали и согласны с пользовательским соглашением http://www.mathnet.ru/rus/agreement

Параметры загрузки:

IP: 54.166 .219 .16

26 апреля 2023 г., 08:54:19 
УДК 512.7

\title{
О связи задач диагностического и проверяющего тестирования бесповторных функций
}

\author{
() 2011 г. Д. В. Чистиков
}

\begin{abstract}
Рассматривается задача построения условного диагностического теста для бесповторных функций в произвольном базисе с помощью запросов значения функции в точке и запросов проверки произвольной подфункции на тождественное равенство постоянной. Устанавливается связь этой задачи с родственной задачей проверяющего тестирования, доказывается ее полиномиальная разрешимость для широкого класса булевых функций.

Работа выполнена при поддержке ФЦП «Научные и научно-педагогические кадры инновационной России» на 2009-2013 г.
\end{abstract}

Рассмотрим классическую задачу условного диагностического тестирования (расшифровки) для булевых функций, представимых формулами над множеством $\mathfrak{B}$ без повторения символов переменных, бесповторных в базисе $\mathfrak{B}$ функций. Требуется построить алгоритм, который, задавая вопросы о значениях неизвестной (находящейся в черном ящике) бесповторной в $\mathfrak{B}$ функции $f\left(x_{1}, \ldots, x_{n}\right)$, идентифицирует эту функцию. Под сложностью алгоритма при этом понимается задаваемое им число вопросов в худшем случае.

Предполагается, что алгоритмы обладают возможностью получать правильные ответы на вопросы следующих естественных типов:

(1) каково значение $f$ на наборе $\left(\alpha_{1}, \ldots, \alpha_{n}\right) \in\{0,1)^{n}$ ?

(2) однозначно ли определено значение $f$, если известны лишь значения некоторых переменных $\alpha_{i_{1}}, \ldots, \alpha_{i_{k}} \in\{0,1\}$ ?

Показывается, что в такой постановке задача диагностического тестирования для бесповторных функций является невырожденной и полиномиально разрешимой (относительно числа переменных $n$ неизвестной функции) для широкого класс базисов, включая элементарный. Устанавливается, что достаточным условием полиномиальной разрешимости в случае произвольного конечного базиса $\mathfrak{B}$ является полиномиальная разрешимость (в смысле функции Шеннона) соответствующей задачи проверяющего тестирования с использованием вопросов только типа (1).

Сделаем вначале несколько замечаний по поводу задачи диагностики с использованием только запросов значения функции в точке. Уже в случае элементарного базиса $\{\&, \vee, \neg\}$ такая постановка оказывается вырожденной: чтобы определить, какая из конъюнкций $n$ переменных находится в черном ящике, требуется задать (в худшем случае) не 
менее $2^{n}-1$ вопросов. Уход от рассмотрения немонотонных функций приводит к базису $\{\&, \vee\}$, для которого алгоритм расшифровки сложности $O\left(n^{2}\right)$ был построен в [1].

Одна из невырожденных постановок для случая произвольного базиса изучалась в рамках вычислительной теории обучения (computational learning theory). Использовалась классическая модель Д. Англуин [2], расширяющая возможности распознающего функцию алгоритма и позволяющая выполнять (в дополнение к запросам значения функции в точке - точечным запросам) запросы эквивалентности, то есть вопросы вида: верно ли, что неизвестная функция выражается бесповторной (в данном случае) формулой $\mathscr{\text { ? }}$ Ответ на этот запрос - либо да, либо контрпример (такой входной набор $x$, что $f(x) \neq g(x)$, где $g-$ функция, реализуемая формулой ()

Отметим, что возможная интерпретация различных видов запросов заключается в том, что алгоритм расшифровки может взаимодействовать с одним или несколькими оракулами - специальными агентами, каждый из которых обладает возможностью отвечать на вопросы одного конкретного типа. Данная трактовка восходит к классической работе [4].

Другой подход к построению невырожденной постановки, также связанный с расширением доступного алгоритму арсенала запросов, предполагает обращение к подкубам булева куба $\{0,1\}^{n}$ или, что то же самое, к подфункциям неизвестной функции $f$. В работе [5] изучались возможности алгоритмов, запрашивающих сумму по модулю 2 всех значений функции на произвольном подкубе. Такие запросы, в отличие от запросов эквивалентности, являются обобщением стандартных запросов значения функции в точке. В работе показано, что при наличии информации о существенности переменных $f$ в случае элементарного базиса $\{\&, \vee, \neg\}$ для диагностики достаточно $n^{2}-n+1$ запросов, причем любое расширение базиса приводит к экспоненциально сложной постановке. Принципиальная необходимость использования информации о существенности переменных обоснована в работе [6], где доказано, что отсутствие такого требования также приводит к задаче экспоненциальной сложности.

В работе [6] предложены также другие варианты обобщения стандартных точечных запросов, один из которых (с точностью до переобозначений) и рассматривается в настоящей статье. Этот вопрос в случае ненулевой размерности возвращает 1 , если $f$ в данном подкубе тождественно равна некоторой константе, и 0 в противном случае. Пара запросов, эквивалентная запросу данного типа, вводилась еще в работе [4]. Определенные так запросы естественно называть запросами тождественности: они дают возможность получать ответы на вопросы определенных выше типов 1 и 2.

В настоящей статье доказывается, что для произвольного конечного базиса достаточным условием полиномиальной разрешимости задачи условного диагностического тестирования с запросами тождественности является полиномиальная разрешимость родственной задачи проверяющего тестирования. Особо отметим, что в отличие от описанной выше похожей постановки с запросами суммы по модулю 2 априорной информации о существенности переменных при этом не требуется. Предлагаемое доказательство основывается на полиномиальном алгоритме из [3], использующем точечные запросы и запросы эквивалентности, логически это означает, что существенным образом используется также полиномиальная разрешимость соответствующей диагностики для произвольного конечного базиса в классической модели Англуин.

Невырожденная постановка задачи проверяющего тестирования для бесповторных функций - тестирование относительно бесповторной альтернативы - была впервые описана в работе А. А. Вороненко [7]: для бесповторной в базисе $\mathfrak{B}$ функции $f\left(x_{1}, \ldots, x_{n}\right)$, существенно зависящей от всех своих переменных, требуется построить проверяющий тест - множество наборов, отличающих $f$ от всех остальных бесповторных в $\mathfrak{B}$ функций тех же переменных. Отметим, что и здесь невырожденность определяется наличием 
априорной информации о существенности переменных функции $f$. Данная постановка исследовалась в различных базисах, причем изучались как тесты для индивидуальных функций, так и подходы к определению значений функции Шеннона $C_{\mathfrak{B}}(n)-$ минимального числа наборов, достаточного для тестирования (проверки) всякой бесповторной функции $n$ переменных.

В настоящей статье устанавливается специальная верхняя оценка величины $D_{\mathfrak{B}}(n)$ - минимальной сложности условного диагностического теста (алгоритма расшифровки) для бесповторных в базисе $\mathfrak{B}$ функций $n$ переменных. Под сложностью при этом понимается выполняемое алгоритмом число запросов в худшем случае, причем доступными являются только запросы тождественности. Основной результат формулируется следующим образом.

Теорема 1. Для любого конечного базиса $\mathfrak{B}$ величины $C_{\mathfrak{B}}(n)$ и $D_{\mathfrak{B}}(n)$ связаны соотношением

$$
D_{\mathfrak{B}}(n) \leqslant 2 n C_{\mathfrak{B}}(n)+O\left(n^{l+2}\right),
$$

где l - максимальная арность функиий из $\mathfrak{B}$.

Ясно, что если $C_{\mathfrak{B}}(n)$ растет не быстрее некоторого полинома от $n$, то и $D_{\mathfrak{B}}(n)$ ограничена полиномом, то есть рассматриваемая задача условного диагностического тестирования полиномиально разрешима. Опишем известные достаточные условия полиномиальности функции Шеннона для задачи проверяющего тестирования. В $[7,8]$ разработан метод, позволяющий восстанавливать бесповторную функцию по ее значениям на набоpax множества гиперкубов существенности - специального множества мощности $O\left(n^{l}\right)$, где $n$ - число переменных восстанавливаемой (тестируемой) функции, $l$ - максимальная арность функции из $\mathfrak{B}$. Показано, что множество гиперкубов существенности является проверяющим тестом для всякой функции, выражаемой бесповторной формулой над $\mathfrak{B}$ без символов так называемых дискриминирующих функций арности $l-2$ и менее (функция переменных $X$ называется дискриминирующей, если найдется такое собственное подмножество $X^{\prime} \subset X, X^{\prime} \neq X$, что каждая из остаточных подфункций переменных $X^{\prime}$ имеет хотя бы одну фиктивную переменную). Из последнего факта и того, что все дискриминирующие функции существенно зависят от трех и более переменных, следует, что если базис $\mathfrak{B}$ состоит только из функций арности $l \leqslant 4$, то метод гиперкубов существенности дает проверяющие тесты длины $O\left(n^{l}\right)$ для всех бесповторных в $\mathfrak{B}$ функций. В работе [9] этот результат распространен на случай произвольного базиса с $l=5$. Предположение о справедливости этого факта для любого конечного базиса $\mathfrak{B}$ было названо гипотезой гиперкуба. Выполнение этой гипотезы для базиса $\mathfrak{B}$, не содержащего функций арности более $l$, означает, в частности, что $C_{\mathfrak{B}}=O\left(n^{l}\right)$. Таким образом, на данный момент можно говорить о том, что все условия сформулированной выше теоремы выполнены, например, в случаях, описываемых следующим утверждением.

Следствие 1. Пусть $\mathfrak{B}$ - конечный базис, не содержащий либо дискриминирующих функциий, либо функций арности 6 и более. Тогда бесповторные в $\mathfrak{B}$ функции допускают расшифровку полиномиальным числом запросов тождественности.

Перейдем теперь к доказательству анонсированного результата. Сформулируем и докажем лемму о моделированию запроса эквивалентности с помощью запросов тождественности.

Лемма 1. Для любого конечного базиса $\mathfrak{B}$ произвольный запрос эквивалентности моделируется не более, чем $2 C_{\mathfrak{B}}(n)+O(n)$ запросами тождественности. 
Доказательство. Пусть $f\left(x_{1}, \ldots, x_{n}\right)$ - функция, находящаяся в черном ящике, а $g-$ функция, реализуемая формулой $\mathscr{G}$ - параметром запроса эквивалентности. Для моделирования необходимо установить, выполняется ли соотношение $f \equiv g$, и если это так, возвратить ответ «да», в противном случае предоставить контрпример $y$ - такой входной набор, что $f(y) \neq g(y)$.

Воспользуемся тем, что функция $g$ бесповторна в базисе $\mathfrak{B}$. Обозначим символом $g^{\prime}$ функцию, получаемую из $g$ удалением всех ее фиктивных переменных. Согласно определению функции Шеннона, $g^{\prime}$ допускает проверяющий тест $T^{\prime}$ длины, (мощности) не более $C_{\mathfrak{B}}(n)$. Зафиксируем произвольные значения фиктивных переменных $g$ и, расширив наборы теста $T^{\prime}$ этими значениями, получим некоторую таблицу $T$ точечных запросов. Продемонстрируем, как можно использовать $T$ для моделирования запроса эквивалентности.

Выполним вначале точечные запросы для каждого набора из $T$. Если при какомлибо $x$ результат запроса $f(x)$ не совпадает с $g(x)$, то $x$ - искомый контрпример и моделирование завершается. В противном случае, поскольку $T^{\prime}-$ проверяющий тест, мы приходим к выводу, что функция $g^{\prime}$ совпадает с соответствующей подфункцией $f^{\prime}$ функции $f$. Рассмотрим этот случай подробнее.

Необходимо проверить справедливость равенства $f(x)=g(x)$ для всех $x$. Для каждого набора $x^{\prime}$ из $T^{\prime}$ выполним запрос тождественности, обращенный к подкубу, задаваемому этим набором (существенные переменные $g$ фиксируются значениями из $x^{\prime}$, фиктивные остаются свободными). Если все такие запросы возвратят 1, то все соответствующие подфункции - константы. Таким образом, в этом случае $g^{\prime}$ совпадает со всевозможными подфункциями функции $f$, получающимися подстановками констант на места всех фиктивных переменных $g$, поэтому моделирование завершается ответом «да». Заметим, что до сих пор было выполнено не более чем $2 C_{\mathfrak{B}}(n)$ запросов.

Предположим теперь, что запрос тождественности для некоторого подкуба возвратил 0. Найдем в этом случае такой набор $y$, что $f(y) \neq g(y)$. Если обнаруженный нами подкуб с неконстантной остаточной подфункцией имеет размерность 1 , то для поиска $y$ достаточно одного точечного запроса. В противном случае поделим этот подкуб на два подкуба меньшей размерности и выполним запросы к этим подкубам. Если оба эти запроса вернут 1, то запрос значения $f$ на любом из этих подкубов определит, какой из них целиком состоит из искомых контрпримеров. Если же хотя бы один из запросов к этим двум подкубам вернет 0, то продолжим процесс деления пополам для этого подкуба. Нетрудно видеть, что вся процедура поиска $y$ требует $O(n)$ запросов, а моделирование в целом - не более $2 C_{\mathfrak{B}}(n)+O(n)$ запросов, что и требовалось доказать.

Из леммы, в частности, следует, что если для конечного базиса $\mathfrak{B}$ верна гипотеза гиперкуба, то запрос эквивалентности можно промоделировать $O\left(n^{l}\right)$ запросами тождественности, где $l$ - максимальная арность функций из $\mathfrak{B}$. Отметим, что в вырожденном случае $l=0$, так что запрос эквивалентности моделируется одним точечным запросом. Докажем, используя лемму, сформулированную выше теорему.

Доказательство теоремы 1. Применение леммы к алгоритму расшифровки из [3], использующему $O\left(n^{l+2}\right)$ точечных запросов и $n$ запросов эквивалентности, дает необходимый алгоритм. Число выполненных запросов не превосходит величины

$$
n\left(2 C_{\mathfrak{B}}(n)+O(n)\right)+O\left(n^{l+2}\right)=2 n\left(C_{\mathfrak{B}}(n)+O\left(n^{l+2}\right)\right),
$$

и из подкубов ненулевой размерности обращаются не более, чем к $n\left(C_{\mathfrak{B}}(n)+O\left(n^{2}\right)\right)$ из них. Теорема доказана. 
Итак, если $\mathfrak{B}$ - конечный базис, для которого верна гипотеза гиперкуба, то задача условного диагностического тестирования (расшифровки) для бесповторных в $\mathfrak{B}$ функций допускает решение алгоритмом, выполняющим $O\left(n^{l+2}\right)$ запросов тождественности, где $l$ - максимальная арность функций их $\mathfrak{B}$, что и приводит нас к утверждению следствия. В общем случае, если $C_{\mathfrak{B}(n)}(n)=O\left(n^{k}\right)$, то справедливо соотношение $D_{\mathfrak{B}}(n)=O\left(n^{\max \{k+1, l+2\}}\right)$, а к подкубам нулевой размерности обращается не более $O^{\max \{k+1,2\}}$ запросов.

В заключение отметим, что мы могли бы определить запрос тождественности не как обобщение точечного запроса, а несколько иначе: положить возвращаемое значение равным 1 для любого подкуба размерности 0 (точки булева куба). В этом случае естественно было бы разрешить алгоритмам использовать также стандартные запросы значения функции в точке, при этом техника моделирования осталась бы прежней, а основные полученные результаты сохранили бы справедливость с соответствующими уточнениями формулировок.

\section{Список литературы}

1. Angluin D., Hellerstein L., Karpinski M., Learning read-once functions with queries. J. ASM (1993) 40, 185-210.

2. Angluin D., Queries and concept learning. Machine Learning (1987) 2, 319-342.

3. Bshouty N. H., Hancock T. R., Hellerstein L., Learning Boolean read-once formulas over generalized bases. J. Comput. Syst. Sci. (1995) 50, №3, 521-542.

4. Valiant L. G., A theory of the learnable. Comm. ACM (1984) 27, 1134-1142.

5. Вороненко А. А., Чистиков Д. В., Расшифровка бесповторных функций оракулом - счетчиком четности. Прикладная математика и информатика (2010) 34, 93-106.

6. Вороненко А. А., О задачах глобального тестирования (расшифровки) бесповторных булевых функций. В сб.: Материаль ІІІ Российской школь-семинара «Синтаксис и семантика логических систем». Изд-во ГОУ ВПО «Восточно-Сибирская государственная академия образования», Иркутск, 2010, с. 17-22.

7. Вороненко А. А., О проверяющих тестах для бесповторных функций. Математические вопросы кибернетики (2002) 11, 163-176.

8. Вороненко А. А., Распознавание бесповторности в произвольном базисе. Прикладная математика и информатика (2006) 23, 67-84.

9. Вороненко А. А., Чистиков Д. В., О тестировании бесповторных булевых функций в базисе $B_{5}$. В сб.: Материаль ХVII Международной школь-семинара «Синтез и сложность управляюших систем». ИМСО РАН, Новосибирск, 2008, с. 24-30.

Статья поступила 15.11.2010. 